\title{
Avaliação das compensações linfáticas no pós-operatório de câncer de mama com dissecção axilar através da linfocintilografia
}

\author{
Evaluation of lymphatic compensation by lymphoscintigraphy in the \\ postoperative period of breast cancer surgery with axillary dissection
}

\begin{abstract}
Laura Ferreira de Rezende ${ }^{1}$, Felipe Villela Pedras Resumo

O sistema linfático é um componente do corpo humano intimamente relacionado ao sistema venoso. Entretanto, o conhecimento científico a seu respeito é limitado. A etiologia e os fatores de risco para o desenvolvimento do linfedema no pós-operatório de câncer de mama são multifatoriais e ainda não foram completamente esclarecidos. O objetivo desta revisão da literatura foi descrever o padrão linfocintilográfico e avaliar as compensações linfáticas do membro superior no pós-operatório de câncer de mama com dissecção axilar.
\end{abstract}

Palavras-chave: Sistema linfático, drenagem linfática, dissecção axilar, linfocintilografia, câncer de mama.

\section{Introdução}

O câncer de mama apresenta grandes e significativos índices de mortalidade entre as mulheres, sendo o segundo tipo de câncer mais freqüente no mundo e o mais comum entre a população feminina. No Brasil, para o ano de 2008, são esperados 49.400 novos casos, com uma taxa bruta estimada de 51 casos para cada 100.000 mulheres $^{1}$.

De acordo com a American Cancer Society, existem hoje nos Estados Unidos entre 1 e 2 milhões de sobreviventes ao câncer de mama, e cerca de 15 a 20\% delas convivem diariamente com algum desconforto ou incapacidade em seus membros superiores. Estima-se que

\begin{abstract}
The lymphatic system is a component of the human body that is closely related to the venous system. However, scientific knowledge of this system is limited. The etiology and risk factors for the development of postoperative lymphedema in patients with breast cancer seem to be multifactorial and have not been fully understood yet. The objective of this review of the literature was to describe lymphoscintigraphic pattern and to evaluate upper limb lymphatic compensation following breast cancer surgery with axillary dissection.
\end{abstract}

Keywords: Lymphatic system, lymphatic drainage, axillary dissection, lymphoscintigraphy, breast cancer.

entre 120.000 e 600.000 pacientes sofram de alguma complicação pós-operatória ${ }^{2}$.

$\mathrm{O}$ linfedema de membro superior ipsilateral à mama operada, complicação com tempo de instalação variável entre 2 e 92 meses, com média de 14 meses $^{3,4}$, apresenta incidência entre 24 e $49 \%$ após mastectomia, entre 4 e $28 \%$ após tumorectomia com dissecção axilar ${ }^{5}$ e $34 \%$ após cirurgia combinada à radioterapia ${ }^{6}$, promovendo um substancial prejuízo funcional e psicológico para a paciente.

O linfedema é a complicação pós-operatória mais comum, e seus efeitos adversos afetam diretamente a qualidade de vida das pacientes. Embora sua incidência esteja diminuindo devido ao diagnóstico precoce e ao

1. Doutora. Fisioterapeuta, Departamento de Tocoginecologia, Faculdade de Ciências Médicas, Universidade Estadual de Campinas (UNICAMP), Campinas, SP.

2. Médico. Serviço de Medicina Nuclear, Departamento de Radiologia, Faculdade de Ciências Médicas, UNICAMP, Campinas, SP.

3. Doutor. Serviço de Medicina Nuclear, Departamento de Radiologia, Faculdade de Ciências Médicas, UNICAMP, Campinas, SP.

4. Doutora. Departamento de Tocoginecologia, Faculdade de Ciências Médicas, UNICAMP, Campinas, SP.

Não foram declarados conflitos de interesse associados à publicação deste artigo.

Artigo submetido em 17.07.08, aceito em 14.10.08. 
progresso nas estratégias terapêuticas - em especial à técnica da biópsia do linfonodo sentinela -, o linfedema ainda permanece como um desafio significativo para as pacientes e seus fisioterapeutas ${ }^{2}$. Szuba et al. ${ }^{7}$ acreditam que, apesar desses avanços, o aumento da incidência de câncer de mama e o aumento de sobrevida das pacientes levarão ao conseqüente aumento da incidência do linfedema.

O linfedema é uma doença crônica, progressiva e geralmente incurável. O aumento do volume do membro pode desfigurar a imagem corporal, assim como aumentar a morbidade física e psicológica da paciente, além de promover significativo prejuízo na função ${ }^{5}$. É uma alteração de difícil tratamento, e a contínua busca por melhores resultados não justifica a existência de tratamentos insatisfatórios, embora esses resultados freqüentemente não apresentem respostas ideais que atendam às expectativas das pacientes e de seus terapeutas $^{8}$.

A etiologia e os fatores de risco para o desenvolvimento do linfedema em pacientes submetidas à cirurgia por câncer de mama parecem ser multifatoriais e ainda não completamente compreendidos. O risco para o aparecimento do linfedema está associado à dissecção e radioterapia axilar, obesidade, extensão da técnica cirúrgica, infecção ${ }^{5,9}$, idade, número de linfonodos dissecados, número de linfonodos positivos e nível de retirada dos linfonodos ${ }^{10}$.

A dissecção axilar, devido à ausência dos linfonodos ou apenas com a preseça de linfonodos remanescentes, leva à redução da capacidade de transporte de linfa de um sistema vascular linfático intacto de tributárias, isto é, haverá uma redução do transporte de linfa no braço e no quadrante superior do tronco ${ }^{11}$.

O sistema linfático possui várias funções importantes. Dentre elas, o controle da homeostase macromolecular, absorção de lipídeos, metástases, função imunológica e controle dos fluidos teciduais ${ }^{12}$. Tem como principal característica a capacidade de remover líquidos e proteínas dos espaços intersticiais. A remoção desses elementos, por sua vez, só é possível através da membrana capilar linfática, que é mais permeável que a membrana capilar sanguínea. Dessa forma, quando ocorre a falência do sistema linfático, associada à inadequada ação dos macrófagos e conseqüente estagnação de proteínas plasmáticas, pode-se observar o surgimento do linfedema ${ }^{13}$.

Após a dissecção axilar, o sistema linfático buscará mecanismos de compensação na tentativa de suprir a ausência dos linfonodos retirados, adequando, assim, a capacidade de transporte da linfa. Esses mecanismos de compensação podem ser realizados das seguintes formas ${ }^{11}$ :

- Os vasos linfáticos íntegros remanescentes da cirurgia e da radioterapia iniciam suas funções valvulares;

- Surge atividade de fluxos linfáticos colaterais com anastomoses axilo-axilares e axilo-inguinais;

- Através dos canais de tecido conjuntivo e do plexo superficial linfático da derme não-valvulado, tecidos ricos em proteínas migram para fora da área de estase linfática, em direção aos limites do quadrante superior do tronco, promovendo vasos linfáticos saudáveis;

- Anastomoses linfolinfáticas se desenvolvem na área de ressecção axilar;

- Anastomoses linfovenosas periféricas podem estabilizar a capacidade de transporte de linfa através de novas conexões entre veias e linfáticos;

- Macrófagos provenientes do maior número possível de capilares sanguíneos da região da estase linfática iniciam a lise de proteínas, com o objetivo de estagnar a concentração de proteínas plasmáticas.

Em estudo com o cadáver de uma mulher com 81 anos e história de mastectomia com dissecção axilar direita há 11 anos, observou-se completa ausência de caminhos linfáticos superficiais no braço direito e próximo ao cotovelo, encontrando fibrose e bloqueio dos canais linfáticos. Foram identificadas várias formas de compensação linfática: refluxo dérmico; anastomoses linfolinfáticas superficiais; anastomoses linfolinfáticas superficiais e profundas; atrofia do vaso linfático significando bloqueio; e anastomoses linfovenosas ${ }^{14}$.

O linfedema é um problema quantitativo entre o fluxo linfático produzido e a capacidade de transporte. Se os mecanismos de compensação forem insuficientes, 
o equilíbrio entre a produção e o transporte estará arruinado; se a produção normal de linfa for maior que a capacidade de transporte, o linfedema aparecerá imediatamente ${ }^{11}$.

Quando um linfonodo é ressecado, ocorre um processo de linfangiogênese e, em muitos casos, a capacidade de transporte dos novos vasos formados parece suficiente para prevenir a manifestação clínica do edema. A questão é conhecer se os parâmetros normais acompanham ou não tal processo. Essa resposta pode ser importante, sendo presumível que qualquer alteração sutil de transporte linfático pode alterar a drenagem linfática do tecido e, conseqüentemente, aumentar o risco de desenvolvimento do linfedema ${ }^{15}$.

Em um estudo experimental com animais, foi possível observar que após a retirada do linfonodo, em um período de 4 semanas houve o aparecimento de um plexo de pequenos vasos como uma ponte lacunada de ligação entre o ducto pré-nodal e os vasos pré-nodais, sem evidência clínica de edema no membro do animal, sugerindo que a drenagem linfática superficial tinha sido restaurada. Entretanto, essas pontes apresentaram estruturas muito irregulares, o que gerava um aumento da resistência do fluxo linfático, provocando uma relação não-linear entre o fluxo e a pressão de perfusão. Pode-se notar, também, que tais estruturas se enredaram no tecido fibroso, distorcendo, assim, o caminho do vaso linfático e contribuindo para o aumento da resistência do fluxo. Dessa forma, apesar do linfonodo removido promover a geração de novos vasos linfáticos, houve um prejuízo para o fluxo de transporte da linfa ${ }^{15}$.

A regeneração dos vasos linfáticos interrompidos através das anastomoses linfolinfáticas é sensível à alterada formação cicatricial, seroma pós-operatório, radioterapia e exercícios precoces inadequados para a reabilitação do ombro ${ }^{11}$, sendo fatores de prejuízo a esse processo de neoformação. Tecidos menos prejudicados em cirurgias menos agressivas permitem novas conexões linfolinfáticas ${ }^{16}$.

A restauração do fluxo linfático é beneficiada pela boa cicatrização tecidual, pela imobilização do ombro ipsilateral à cirurgia, pela drenagem linfática manual e pela contração muscular ${ }^{11,12,16}$.

\section{Linfocintilografia}

A linfocintilografia é atualmente defendida como o principal teste diagnóstico para o sistema linfático periférico, permitindo a visualização de vasos linfáticos e linfonodos, bem como a quantificação do transporte linfático. São considerados fluxos anormais do sistema linfático a falta da trilha de migração e a lentidão no transporte do radiofármaco, o fluxo contrário do fluido para a derme, a presença de largos vasos linfáticos, a não-visualização dos vasos linfáticos do lado envolvido e a ausência ou pobre visualização dos linfonodos remanescentes e/ou detectáveis ${ }^{17,18}$.

A linfocintilografia apresenta aplicação clínica para indicar e quantificar a drenagem linfática sob os pontos de vista morfológico e funcional, determinar o número de linfonodos sentinelas e para identificar pacientes de risco para desenvolvimento de linfedema após a dissecção linfonodal ${ }^{7}$. É um método confiável para mensurar o fluxo linfático em condições incertas de aumento ou redução dessas atividades ${ }^{19,20}$. A interpretação visual da imagem da linfocintilografia associada ao tempo de aparecimento do radiofármaco na região dos linfonodos promove informações confiáveis sobre a normalidade ou não do sistema linfático, com especificidade de $100 \%$ e sensibilidade de 92 a 97\% ${ }^{17,21}$. Para análise das imagens, são estudadas as características de distribuição das partículas vistas durante a linfocintilografia, sendo considerados sinais de presença de linfedema o acúmulo do radiofármaco no tecido, presença de refluxo dérmico, linfangectasia, interrupção de vasos linfáticos e demora no transporte ${ }^{22,23}$.

O protocolo de linfocintilografia não é padronizado e difere de acordo com o serviço de diagnóstico. Diferenças incluem a escolha do radiofármaco, o tipo e o local da injeção, o uso de avaliações estáticas ou dinâmicas, e o número e tempo entre essas avaliações ${ }^{22}$.

O primeiro radiofármaco aprovado pelo Food and Drug Administration (FDA), e não mais utilizado, foi um colóide de baixo tamanho (3 a $30 \mathrm{~nm}$ ), marcado com pertecnetato- ${ }^{99 \mathrm{~m}} \mathrm{Tc}$. Depois surgiu o enxofre coloidal- ${ }^{99 \mathrm{~m}} \mathrm{Tc}(10$ a $1.000 \mathrm{~nm})$, que pode ser filtrado para remover grandes partículas, criando um tamanho de partícula uniforme $(10-50 \mathrm{~nm})^{22}$. 
Outro microcolóide freqüentemente utilizado é a albumina, uma partícula homogênea no tamanho (95\% inferior a $80 \mathrm{~nm}$ ) e de fácil marcação. É rapidamente visualizada após a injeção, indolor para o paciente, e adequada para estudos quantitativos. É descrita também a utilização de partículas como a albumina sérica humana, o dextran e a imunoglobulina humana marcados com ${ }^{99 \mathrm{~m}} \mathrm{Tc}$. Esses podem ser visualizados através da linfocintilografia por dois mecanismos, a reabsorção e o transporte da partícula para dentro do capilar linfáti$\mathrm{co}^{22}$. No Brasil, o dextran (6-7 nm) é o mais utilizado, sendo adequado para o diagnóstico de linfedema. Todos os radiofármacos são marcados pelo ${ }^{99 \mathrm{~m}} \mathrm{Tc}$.

O dextran é um radiofármaco solúvel na linfa, e tem suas moléculas grandes o suficiente para não penetrar pela membrana do capilar sanguíneo após sua injeção no interstício. A filtração glomerular e a excreção urinária ocorrem quando o dextran pesar menos do que 40 $\mathrm{kDa}$ após a sua injeção no interstício ${ }^{24}$.

A técnica de injeção do radiofármaco para a realização da linfocintilografia é outro ponto discutido. Para estudo do extravasamento linfático, a injeção subcutânea é melhor que a intradérmica. Para estudos da superfície linfática nas extremidades, podem ser utilizadas as vias subcutâneas ou intradérmicas ${ }^{22}$.

A interpretação da imagem da linfocintilografia é melhor realizada com base no reconhecimento da distribuição anormal do radiofármaco e no conhecimento do tempo relativo que esse demora para alcançar os linfonodos regionais ${ }^{17}$. Bourgeois et al. ${ }^{19}$ preconizam, dentre os parâmetros da linfocintilografia, a utilização do tempo que o radiofármaco demora a alcançar os linfonodos axilares e Szuba et al. ${ }^{7}$ a quantidade de acúmulo do radiofármaco nos linfonodos axilares.

Cambria et al. ${ }^{25}$ propuseram a avaliação do transporte linfático através da linfocintilografia dinâmica pela análise do tempo de aparecimento do radiofármaco no linfonodo Essa escala considerava também o transporte do movimento linfático, a distribuição dos caminhos linfáticos, a visualização dos linfonodos e dos vasos linfáticos.
O’Mahony et al. ${ }^{26}$ propuseram a classificação de acordo com a qualidade da imagem, respeitando a definição dos vasos linfáticos em muito pobre, pobre, adequado, bom e excelente. Szuba et al. ${ }^{27}$ desenvolveram uma escala empírica de linfocintilografia estática para visualização dos linfonodos axilares, considerando o padrão normal como linfonodos visíveis e simétricos e a pior anormalidade como linfonodos não visíveis. Gloviczki et al. ${ }^{21}$ classificaram o tempo de aparecimento do radiofármaco nos linfonodos combinado com o grau de visualização em claramente visível, pouco visível e não visível.

O estudo linfocintilográfico é considerado normal se discretos canais linfáticos drenarem a extremidade do membro e se os linfonodos regionais forem visualizados em até 1 hora. Tempo semelhante é considerado estudo linfocintilográfico normal para a chegada do radiofármaco no fígado ${ }^{28}$. O'Mahony et al. ${ }^{26}$ e Scarbrook et al. ${ }^{23}$ consideraram 30 minutos o tempo normal de chegada do radiofármaco nos linfonodos regionais. A imagem do fígado pode ser visualizada quando o radiofármaco cair na corrente sanguínea. Dessa maneira, espera-se a presença do radiofármaco em imagens tardias, uma vez que a presença precoce sugere injeção intravascular ${ }^{23}$.

Lane et al. ${ }^{29}$ realizaram estudos linfocintilográficos de 10 em 10 minutos até completar 1 hora. Weissleder \& Weissleder ${ }^{30}$ consideraram normal que em 10 minutos o radiofármaco apareça nos linfonodos. Gloviczki et al. ${ }^{21}$ também estabeleceram 10 minutos como tempo normal para o transporte do radiofármaco, considerando 1 hora como transporte atrasado e 2 horas como transporte seriamente comprometido.

Para referência de normalidade do padrão de drenagem linfática, sugere-se o controle com o membro superior contralateral ou controle na população em geral ${ }^{19}$. O grupo avaliado com linfocintilografia no pósoperatório sem o desenvolvimento de linfedema apresenta o estado de funcionalidade similar ao do grupo-controle ${ }^{29}$.

A linfocintilografia antes e após a dissecção axilar tem um potencial de oferecer informações clínicas para predizer o desenvolvimento de alterações linfáticas que 
vão culminar no linfedema ${ }^{19,26,27,31}$. Investigar o membro superior ipsilateral quanto à dissecção axilar no pósoperatório sem desenvolvimento de linfedema é uma proposta de Bourgeois et al. ${ }^{19}$. Pouco se conhece sobre o estudo linfocintilográfico pré-operatório. Acredita-se que alterações anatômicas e funcionais já presentes previamente à cirurgia teriam importância no desenvolvimento do linfedema pós-dissecção axilar. Em um único estudo é relatado 7,5\% de anormalidade do sistema linfático no pré-operatório, sendo que $85 \%$ dessas anormalidades desenvolveram linfedema em um período aproximado de 34 meses $^{19}$.

Baulieu et al. ${ }^{32}$ avaliaram 32 pacientes com edema no pós-operatório de fratura de tíbia através da linfocintilografia, entre 2 e 10 dias após a cirurgia, sendo que o membro saudável também foi avaliado. Tais pacientes foram divididos em dois grupos, e apenas o grupo com comprometimento linfático desenvolveu linfedema em um período de 3 meses. Dessa forma, os autores puderam inferir que a pré-existência de alterações funcionais do sistema linfático pode justificar o aparecimento precoce do linfedema.

Todos esses resultados são relativos a pacientes que tiveram os três níveis de Berg dissecados e podem ser questionados, uma vez que atualmente a maioria dos cirurgiões disseca os níveis I e II. Entretanto, os linfonodos especificamente relacionados à drenagem do membro superior estão localizados na parte lateral da dissecção axilar, abaixo da veia axilar e acima do segundo nervo intercostal braquial, estando presentes, portanto, no nível I de Berg ${ }^{33}$.

Outro achado importante demonstra que a axila é completamente dissecada em apenas 1/3 das pacientes que foram submetidas à dissecção dos três níveis de Berg, e essa situação depende da experiência do cirurgião. Dessa maneira, a drenagem linfática de membro superior seria interrompida em $29,3 \%$ dos pacientes, considerando-se a ausência de visualização do radiofármaco nos linfonodos axilares através da linfocintilografia ${ }^{19}$.

Em uma análise multivariada, Bourgeois et al. ${ }^{19}$ observaram que três variáveis influenciam, independentemente, o fato dos linfonodos axilares não serem visualizados: a demora da investigação após a cirurgia; a idade da paciente (acima de 60 anos) e o número de linfonodos comprometidos (acima de três) ${ }^{19}$.

A avaliação da função do sistema linfático do membro superior ipsilateral à mama acometida, antes e 60 dias após a cirurgia, permitirá o conhecimento do estado funcional prévio do sistema linfático e a sua repercussão após a dissecção axilar, possibilitando assim a descrição de potenciais fatores preditivos para o desenvolvimento do linfedema. A avaliação prévia dos membros superiores ipsilateral e contralateral permite a detecção de anormalidades clínicas e comparação interna da distribuição linfática, possibilitando uma análise mais fidedigna das alterações do membro superior ipsilateral no pós-operatório ${ }^{23}$. A evidência precoce de tais alterações permitirá o desenvolvimento de ações preventivas, o acompanhamento mais rigoroso dessas pacientes e, possivelmente, a realização precoce do diagnóstico e tratamento.

\section{Referências}

1. Instituto Nacional do Câncer (INCA) [site na Internet]. Brasil, Ministério da Saúde; 2007. [citado 30 dez 2007]. Disponível em: http://www.inca.gov.br.

2. Soran A, D'Angelo G, Begovic M, et al. Breast cancer-related lymphedema: what are the significant predictors and how they affect the severity of lymphedema? Breast J. 2006;12:536-43.

3. Werner RS, McCormick B, Petrek J, Cox L, Cirrincione C, Gray JR. Arm edema in conservatively managed breast cancer: obesity is a major predictive factor. Radiology. 1991;180:177-84.

4. Guedes Neto HI. Arm edema after treatment for breast cancer. Lymphology. 1997;30:35-6.

5. Warren AG, Brorson H, Borud LJ, Slavin SA. Lymphedema: a comprehensive review. Ann Plast Surg. 2007;59:464-72.

6. Lee TS, Kilbreath SL, Refshauge KM, Herbert RD, Beith JM. Prognosis of the upper limb following surgery and radiation for breast cancer. Breast Cancer Res Treat. 2008;110:19-37. Epub 2007 Sep 26.

7. Szuba A, Shin WS, Strauss HW, Rockson S. The third circulation: radionuclide lymphoscintigraphy in the evaluation of lymphedema. J Nucl Med. 2003;44:43-57.

8. Consensus Document of International Society of Lymphology. The diagnosis and treatment of peripheral lymphedema. Lymphology. 2003;36:84-91.

9. Vignes S, Arrault M, Dupuy A. Factors associated with increased breast cancer-related lymphedema volume. Acta Oncol. 2007;46:1138-42.

10. Kiel KD, Rademacker AW. Early stage breast cancer: arm edema after wide excision and breast irradiation. Radiology. 1996;198:279-83. 
11. Foldi E, Foldi M, Clodius L. The lymphedema chaos: a lancet. Ann Plast Surg. 1989;22:505-15.

12. Gashev AA, Zawieja DC. Physiology of human lymphatic contractility: a historical perspective. Lymphology 2001;34:124-34.

13. Guyton AC. Tratado de Fisiologia Medica. Rio de Janeiro: Guanabara Koogan; 1998.

14. Suami H, Pan WR, Taylor GI. Changes in the lymph structure of the upper limb after axillary dissection: radiographic and anatomical study in human cadaver. Plast Reconstr Surg. 2007;120:982-91.

15. Kim C, Li B, Papaiconomou C, Zakharov A, Johnston M. Functional impact of lymphangiogenesis on fluid transport after lymph node excision. Lymphology. 2003;36:111-9.

16. Clodius L. Minimizing secondary arm lymphedema from axillary dissection. Lymphology. 2001;34:106-10.

17. Howarth DM. Increased lymphoscintigraphy flow pattern in the lower extremity under evaluation for lymphedema. Mayo Clin Proc. 1997;72:423-9.

18. Hwang JH, Kwon JY, Lee KW, et al. Changes in lymphatic function after complex physical therapy for lymphedema. Lymphology. 1999;32:15-21.

19. Bourgeois P, Leduc O, Leduc A. Imaging techniques in the management and prevention of posttherapeutic upper limb edemas. Cancer. 1998;83(12 Suppl American):2805-13.

20. Svensson W, Glass DM, Bradley D, Peters AM. Measurement of lymphatic function with technetium-99m-labelled polyclonal immunoglobulin. Eur J Nucl Med. 1999;26:504-10.

21. Gloviczki P, Calcagno D, Schirger A, et al. Noninvasive evaluation of the swollen extremity: experiences with 190 lymphoscintigraphy examinations. J Vasc Surg. 1989;9:683-9; discussion 690.

22. Yuan Z, Chen L, Luo Q, Zhu J, Lu H, Zhu R. The role of radionuclide lymphoscintigraphy in extremity lymphedema. Ann Nucl Med. 2006;20:341-4.

23. Scarsbrook AF, Ganeshan A, Bradley KM. Pearls and pitfalls of radionuclide imaging of the lymphatic system. Part 2: evaluation of extremity lymphoedema. Bri J Radiol. 2007;80:219-26. Epub 2006 May 25.

24. Williams WH, Witte CL, Witte MH, Mcneill GC. Radionuclide lymphangioscintigraphy in the evaluation of peripheral lymphedema. Clin Nucl Med. 2000;25:451-64.
25. Cambria RA, Gloviczki P, Naessens JM, Wahner HW. Noninvasive evaluation of the lymphatic system with lymphoscintigraphy: a prospective, semiquantitative analysis in 386 extremities. J Vasc Surg. 1993;18:773-82.

26. O'Mahony S, Rose SL, Chilvers AJ, et al. Finding an optimal method for imaging lymphatic vessels of the upper limb. Eur J Nucl Med Mol Imaging. 2004;31:555-63.

27. Szuba A, Strauss W, Sirsikar SP, Rockson SG. Quantitative radionuclide lymphoscintigraphy predicts outcome of manual lymphatic therapy in breast cancer-related lymphedema of the upper extremity. Nucl Med Commun. 2002;23:1171-5.

28. Ter SE, Alavi A, Kim CK, Merli G. Lymphoscintigraphy. A reliable test for the diagnosis of lymphedema. Clin Nucl Med. 1993;18:646-54.

29. Lane KN, Dolan LB, Worsley D, Mckenzie DC. Upper extremity lymphatic function at rest and during exercise in breast cancer survivors with and without lymphedema compared with healthy controls. J Appl Physiol. 2007;103:917-25.

30. Weissleder H, Weissleder R. Lymphedema: evaluation of qualitative and quantitative lymphoscintigraphy in 238 patients. Radiology. 1988;167:729-35.

31. Petrek JA, Pressman PI, Smith RA. Lymphedema: current issues in research and management. CA Cancer J Clin. 2000;50:292-307.

32. Baulieu F, Itti R, Taieb W, Richard G, Martinat H, Barsotti J. Lymphoscintigraphy. A predictive test of post-traumatic lymphedema of the lower limbs. Rev Chir Orthop Reparatrice Appar Mot. 1985;71:327-32.

33. Nos C, Lesieur B, Clough KB, Lecuru F. Blue dye injection in the arm in order to conserve the lymphatic drainage of the arm in breast cancer patients requiring an axillary dissection. Ann Surg Oncol. 2007;14:2490-6.

Correspondência:

Laura Ferreira de Rezende Franco

Rua Laguna, 676/43, Jardim dos Estados

CEP 37701-074 - Poços de Caldas, MG

Tel.: (35) 3715.3281

E-mail: laura@fae.br 